\title{
Homocysteine and Lipid Profile in Patients with Coronary Artery Disease
}

\author{
Authors \\ Jayarajan Karumarakkal $^{1}$, Mohan Raj Manjalavil ${ }^{2}$ \\ ${ }^{1}$ Associate Professor, Dept. of Biochemistry, Govt. Medical College, Manjeri, Kerala, India 676121 \\ ${ }^{2}$ Associate Professor, Dept. of Physical Medicine \& Rehabilitation, Govt. Medical College, Kozhikode. \\ Kerala, India 673008 \\ Corresponding Author \\ Jayarajan Karumarakkal
}

Associate Professor, Dept. of Biochemistry, Govt. Medical College, Manjeri Kerala, India 676121

Email: jayaraj1s@gmail.com,Mob-9745303175

\begin{abstract}
Aim of the study: To find out whether there is any significant correlation between concentrations of homocysteine level, hypercholesterolemia, diabetes mellitus, smoking and hypertension in coronary artery disease.
\end{abstract}

Background: Cardiovascular diseases are the major cause of morbidity and mortality worldwide. Diabetes mellitus, obesity, hypercholesterolemia, smoking and hypertension have been recognized as major risk factors for cardiovascular diseases. A new class of emerging risk factor is elevated plasma homocysteine level. This study aims to determine whether there is any significant association between homocysteine lipid profile and other parameters in acute coronary artery disease.

Objectives: 1 . To determine the relation between homocysteine and coronary artery disease.

2. To study the lipid profile and other parameters in patients with coronary artery disease in our population. Settings and Design: Case control study in a tertiary care hospital, Kozhikode, Kerala.

Materials and Methods: Patients in the age group of 20 to 60 years with first attack of acute coronary artery disease of both sexes are included in this study. Sample size was 30 cases and 60 controls. Study period was one year. Levels of homocysteine, fasting lipid profile and other parameters are assessed.

Results: The levels of serum homocysteine, total cholesterol, LDL and fasting blood sugar showed significant increase in cases than in the controls. Triglycerides and HDL levels did not show any associated increase in patients with CAD.

Conclusion: The study reveals homocysteine, diabetes mellitus, hyperlipidemia, hypertension, obesity and smoking has definite role in the generation of coronary artery disease in the patients belonging to the District of Kozhikode.

Key Words: Homocysteine, fasting Lipid profile, coronary artery disease.

\section{Introduction}

Cardiovascular diseases are the major cause of morbidity and mortality worldwide ${ }^{1}$. It is apparently more common in South Asia despite low incidence of several classic coronary risk factors $^{2}$. Diabetes mellitus, obesity, hypercholesterolemia, smoking and hypertension have been recognized as major risk factors for cardiovascular 
diseases; however, they do not fully explain the pathogenesis and causality of these diseases. A new class of emerging risk factor for cardiovascular disease is plasma homocysteine level $^{3}$. This elevated level of homocysteine, a non lipid risk factor for coronary events, acts by promoting thrombosis ${ }^{4}$. Very high levels of this aminoacid is seen in genetically inherited enzyme defects of homocysteine metabolism, which are known to be associated with premature and aggressive vascular disease 5,6. Plasma homocysteine level exceeding $15 \mu \mathrm{mol} / \mathrm{L}$ is termed as hyperhomocysteinemia.

Some studies have shown that up to $10 \%$ of coronary events can be attributed to the increase in homocysteine levels ${ }^{7}$. Additional studies have shown a negative correlation between homocysteine levels and plasma levels of folic acid, vitamin $\mathrm{B} 12$ and $\mathrm{B} 6{ }^{8,9}$. Thus, hyperhomocysteinemia is a new emerging cardiovascular risk factor that could be modified by reducing homocysteine intake or by supplementing the diet with vitamin B12 or B6 ${ }^{10}$.

Atherosclerosis account nearly all coronary artery disease and some proportion of the ischemic strokes. Although the role of cholesterol sub fractions in acute coronary syndromes is well documented, it is still unclear whether the lipid profile plays an important etiologic role in coronary artery disease. Three decades of research in this topic have yielded inconsistent results ${ }^{11,12}$.

Cigarette smokers are more likely than nonsmokers to develop large vessel atherosclerosis as well as small-vessel disease ${ }^{13}$. Diabetic subjects have two or more fold higher risk for CAD compared to non-diabetic population. The prevalence of CAD increased with age and duration of diabetes and nearly $40 \%$ of the subjects with diabetes duration more than 20 years had CAD. Indeed the risk for CAD seemed to increase even at the stage of impaired glucose tolerance $^{14}$.

Heart disease is the most common cause of death in hypertensive patients. Hypertensive heart disease is the result of structural and functional adaptations leading to left ventricular hypertrophy, diastolic dysfunction, CHF, abnormalities of blood flow due to atherosclerotic coronary artery disease and microvascular disease, and cardiac arrhythmias. Individuals with left ventricular hypertrophy are at increased risk for CHD, stroke, CHF, and sudden death.

Obesity also raises blood pressure and blood cholesterol and triglyceride levels, and lowers HDL ("good") cholesterol levels. When increased blood pressure coexists with other risk factors like obesity, smoking, hypercholesterolemia or diabetes, then the risk of heart attack or stroke increases several times. It is in this context we have decided to conduct this study.

\section{Aim of Study}

To determine whether there is any significant association between homocysteine, lipid profile and other parameters in acute coronary artery disease among patients admitted in tertiary care hospital.

\section{Materials and Methods}

Study Design: Case control study

Study Setting: Referral tertiary care hospital, Department of Biochemistry.

Cases: Patients in the age group of 20 to 60 years with first attack of acute coronary artery disease of both sexes, admitted in tertiary care hospital, Kozhikode, Kerala.

Controls: Age and sex matched control without any history of coronary artery disease

Patients in the age group of 20 to 60 years with first attack of acute coronary artery disease of both sexes are included in this study. Sample size was 30 cases and 60 controls. Study period was one year. Levels of Homocysteine, fasting Lipid profile and fasting blood sugar and other parameters are assessed.

\section{Inclusion Criteria}

1) Patients of both sexes in the age group of 20 to 60 years with first attack of acute coronary artery disease admitted in medical wards of Tertiary care hospital. 
2) Those are willing to be included in the study.

3) Patients not on treatment for hypercholestrolemia.

\section{Exclusion Criteria}

1) Patients with repeated attack of coronary artery disease.

2) Patients below 20 and above 60 years.

3) Patients on treatment for Homocystinuria

4) Patients on treatment for hypercholesterolemia

\section{Study Variables}

1) Plasma Homocysteine

2) Fasting Lipid profile

Total cholesterol

Triglycerides

HDL

LDL

Total cholesterol / HDL ratio

3) Fasting blood sugar

\section{Statistical Analysis}

Results were analyzed using SPSS 16 version. T tests are used to analyze the data.

\section{Results}

\section{Plasma Homocysteine}

The main factor estimated was serum homocysteine, which showed significant increase in cases than in the controls, $(p=0.001)$. The details of mean and standard deviation are shown below.

Table -1. Homocysteine

\begin{tabular}{|l|c|c|}
\hline & $\begin{array}{c}\text { Mean (std } \\
\text { deviation) } \\
\text { Case } \mathrm{n}=28\end{array}$ & $\begin{array}{c}\text { Mean (std } \\
\text { deviation) } \\
\text { Control } \mathrm{n}=50\end{array}$ \\
\hline Age & $44.04(9.597)$ & $41.04(9.611)$ \\
\hline Homocysteine & $21.93(10.505)$ & $14.34(4.796)$ \\
\hline
\end{tabular}

Hyperhomocysteinemia frequency - The mean homocysteine level in relation with age group is shown in the chart below. There is an increased homocysteine level in the younger age group.

\section{Hyperhomocysteinemia frequency}

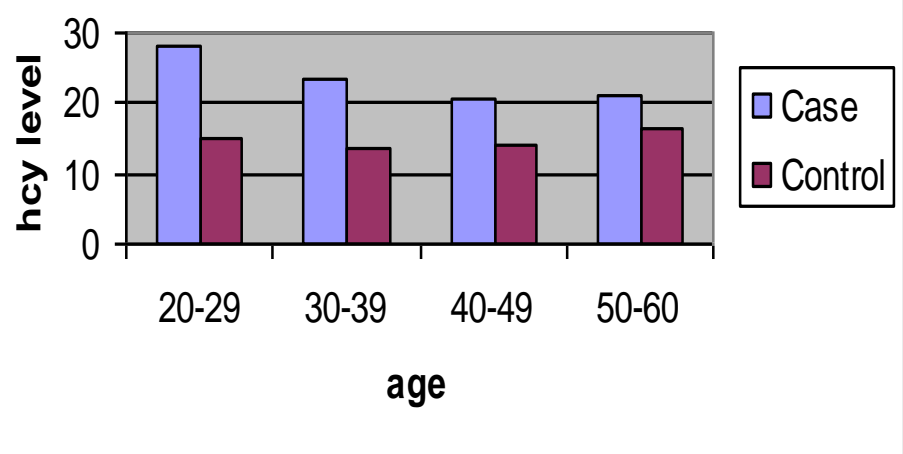

In this study, a statistically significant association was present between coronary artery disease and plasma homocysteine, total cholesterol, LDL, hypertension and waist hip ratio. The association of coronary artery disease with homocysteine and other significant risk factors are shown in the tables below.

\section{Other parameters}

The parameters include FBS, total cholesterol, LDL, blood pressure and waist hip ratio.

\begin{tabular}{|l|c|c|c|}
\hline & $\begin{array}{c}\text { Mean (std } \\
\text { deviation) } \\
\text { Case n=28 }\end{array}$ & $\begin{array}{c}\text { Mean (std } \\
\text { deviation) } \\
\text { Control n=50 }\end{array}$ & $\begin{array}{c}\mathrm{p} \\
\text { value }\end{array}$ \\
\hline FBS & $\begin{array}{c}107.82 \\
(33.450)\end{array}$ & $89.02(20.078)$ & 0.003 \\
\hline Total & $\begin{array}{c}234.43 \\
(35.517)\end{array}$ & $\begin{array}{c}205.04 \\
(40.644)\end{array}$ & 0.001 \\
Cholesterol & $\begin{array}{c}166.64 \\
(26.157)\end{array}$ & $\begin{array}{c}126.26 \\
(28.210)\end{array}$ & 0.001 \\
\hline LDL & $\begin{array}{c}155.79 \\
(14.970)\end{array}$ & $\begin{array}{c}132.80 \\
(20.777)\end{array}$ & 0.001 \\
\hline Systolic BP & $88.79(9.953)$ & $82.88(10.578)$ & 0.018 \\
\hline Diastolic BP & $0.98(0.063)$ & $0.91(0.060)$ & 0.001 \\
\hline WHR & & &
\end{tabular}

\begin{tabular}{|l|c|c|}
\hline variables & $\begin{array}{c}\text { Case } \mathrm{n}=28 \\
\text { Number }(\%)\end{array}$ & $\begin{array}{c}\text { Control } \mathrm{n}=50 \\
\text { Number }(\%)\end{array}$ \\
\hline Homocysteine & $20(70)$ & $23(46)$ \\
\hline FBS & $9(37)$ & $6(12)$ \\
\hline Total Cholesterol & $24(86)$ & $28(56)$ \\
\hline LDL & $26(93)$ & $40(80)$ \\
\hline Hypertension & $21(75)$ & $19(38)$ \\
\hline Obesity-W/H Ratio & $20(71)$ & $10(20)$ \\
\hline
\end{tabular}

\section{Discussion}

The result obtained from this study reveals that hyperhomocysteinemia has definite role in the generation of cardiovascular diseases. The study also reveals that the presence of diabetes and 
hyperlipidemia also is significant in the deveopment of cardiovascular diseases. The $\mathrm{p}$ value obtained for homocysteine was 0.001 and for total cholesterol, LDL and fasting blood sugar were less than 0.05 . The results of the present study are consistent with many case control and prospective studies done earlier.

The quantitative variables like plasma homocysteine, lipid profile and FBS were analyzed by using $\mathrm{T}$ test. In this study the mean value of homocysteine in the cases was 21.93 with a standard deviation of 10.505 and $p$ value was 0.001 . The mean value of homocysteine in the controls was 14.34 with a standard deviation of 4.796. Out of the total 28 cases studied, 20 cases (71\%) had hyperhomocysteinemia. Out of the 50 controls studied only 23 cases (43\%) had hyperhomocysteinemia. So this study proves that homocysteine has a significant association with coronary artery disease in the population studied.

Fasting blood sugar was significantly higher in cases than controls. The mean value of blood sugar in the cases was 107.82 with a standard deviation of 33.450and $\mathrm{p}$ value was 0.003 . The mean value of blood sugar in the controls was 89.02 with a standard deviation of 20.078 .

Out of the total 28 cases studied, 9 cases $(37 \%)$ had increased blood sugar levels. Out of the 50controls studied only 6 cases (12\%) had increased blood sugar levels. So this shows that blood sugar has a significant association with coronary artery disease.

In this study total cholesterol was significantly higher in cases than controls. The mean value of total cholesterol in the cases were 234.43 with a standard deviation of 35.517 and $\mathrm{p}$ value was 0.001 . The mean value of total cholesterol in the controls was 205.04 with a standard deviation of 41.683.

Out of the total 28 cases studied, 24 cases $(86 \%)$ had increased cholesterol levels. Out of the 50controls studied only 28 cases (56\%) had increased cholesterol levels. So this shows that total cholesterol has a significant association with coronary artery disease.
The mean value of LDL in the cases was 166.64 with a standard deviation of 26.157and $p$ value was 0.001 . The mean value of LDL in the controls was 126.26 with a standard deviation of 28.210 . Out of the total 28 cases studied, 26 cases (93\%) had elevated LDL level and out of the 50controls studied only 40 cases (80\%) had elevated LDL level. So this study proves that LDL has a significant association with coronary artery disease.

In this study of the 28 cases, 15 (54\%) were smokers and in the controls, $8(16 \%)$ subjects out of 50 were smokers, and the $\mathrm{p}$ value was 0.001 . So smoking too had a significant association with coronary artery disease in this study.

Thus the present study observes a positive statistical association of the risk factors like homocysteine, diabetes, smoking and hyperlipidemia with coronary artery disease.

\section{Conclusion}

Hyperhomocysteinemia has got a definite independent role in coronary artery disease. The diabetes, and hyperlipidemia also plays a great role in the generation of coronary artery disease in the patients belonging to the District of Kozhikode.

\section{Limitations}

1) This was a hospital based study which did not represent the entire population.

2) Homocysteine levels were compared to controls but a prospective cohort would have been ideal.

3) The correlation of etiological factors like low levels of folates and vitamin B12 in plasma with hyperhomocysteinemia has not come under the inclusion criteria of the present study.

4) The ELISA method was used to estimate homocysteine levels, while high performance liquid chromatography is known to be superior to the above method. 


\section{References}

1. Gilberto Garcia, Juanita Trejos, Beatriz Restrepo, et al : Homocysteine, Folate and Vitamin B12 in Colombian Patients with Coronary Disease, South America Colômbia Universidad Del Patricia Landázuri - Av. Bolivar $12 \mathrm{~N}$ - Armenia (Quindio) Arq Bras Cardiol 2007; 89(2) : 71-76

2. Nigel Unwin, Martin White, Raj Bhopal, Julie Yallop, Louise Walker et al : Heterogeneity of coronary heart disease risk factors in Indian, Pakistani, Bangladeshi, and European origin populations: cross sectional study. BMJ 1999; July 24 319(7204):215-220.

3. Gravina-Taddei CF, Batlouni M, Sarteschi C, Baltar VT, Salvarini NAC, Bertolami $\mathrm{MC}$, et al: Hyperhomocysteinemia as a risk factor for coronary atherosclerotic diseases in the elderly. Arq Bras Cardiol. 2005; 85: 166-73.

4. Kasper, Braunwald, Fauci, Hauser, Longo \& Jameson : Harrison's Principles of Internal Medicine, $17^{\text {th }}$ edition page no 1427-28

5. McCully KS: Vascular pathology of homocysteinemia: implications for the pathogenesis of arteriosclerosis. Am $\mathbf{J}$ Pathol. 1969; 56:111-128.

6. Mudd SH, Skovby F, Levy HL, et al: The natural history of homocystinuria due to cystathionine b- synthase deficiency Am J Hum Genet 1985; 37:1-31

7. Boushey CJ, Beresford SA, Ommenn GS, Motulsky AG: A quantitative assessment of plasma homocysteine as a risk factor for vascular disease: probable benefits of increasing folic acid intakes. JAMA. 1995; 274 (13): 1049-57.

8. Selhub J, Jaques PF, Wilson P, Rush D, Rosenberg IH: Vitamin status and intake as primary determinants of homocysteinemia in and elderly population.JAMA. 1993; 270: 2693-
9. Wald DS, Law M \& Morris JK: Homocysteine and cardiovascular disease: evidence on causality from a metaanalysis. BMJ. 2002; 325: 1202.

10. Schnyder G, Roffi M, Flammer Y: Effect of homocysteine - lowering therapy with folic - acid, vitamin B(12), and Vitamin $\mathrm{B}(6)$ on clinical outcome after percutaneous coronary. Intervention: the Swiss Herat study a randomized controlled trial. JAMA. 2002; 288: 973-9.

11. Dayton S, Chapman JM, Pearce ML, Popjak GJ: Cholesterol, atherosclerosis, ischemic heart disease, andstroke. Ann Intern Med. 1970; 72:97-109.

12. Welin L, Svardsudd K, Wilhelmsen L, Larsson B \& Tibblin G: Analysis of risk factors for stroke in a cohort of men born in 1913. N Engl J Med. 1987; 317:521526.

13. Kasper , Braunwald, Fauci , Hauser, Longo, Jameson Harrison's Principles of Internal Medicine, 17th edition page no 1427-28.

14. R. Deepa, K. Arvind and V. Mohan $\square$ Diabetes and risk factors for coronary arterydisease Madras Diabetes Research Foundation, Gopalapuram, Chennai 600 086, India Current Science, Vol. 83, NO. 12, 25 December 20021497. 